\title{
大对称群 的正则母单位
}

\author{
庄勤 \\ （东北师范大学物理系, 长春）

\section{一、引 亡}

对称群正则母单位在现代物理中,如超核、重子结构、FP系数等问题中应用越来越多. 应 用母单位处理大群乘积约化等问题也是一个有前途的方向. 1962 年的文献 [1] 和 1982 年的 文献 [3] 给出了 $[\lambda]-\left[2^{a} 1^{b}\right] 、\left[4^{a} 3^{b} 2^{c} 1^{d}\right]$ 和 $\left[5^{a} 4^{b} 3^{c} 2^{d} 1^{c}\right]$ 几个较低对称型下的单位表达 式. 表达式中的待定系数 $C_{K}^{[\alpha]}$ 和 $\frac{\theta^{\prime}}{\theta}$ 由一个方程组确定 ${ }^{[3]}$, 而这些方程的系数阵是由文字 $a 、 b$ 、 c...组合而成不能上机, 而手解化简高于 5 元的就几乎不可能. 对称类型越高困难越大.

本文给出一种方法,对任意大的 $N$ 任意高的对称型下的正则母单位都可以直接写出.

\section{二、荊符 $(n-1, n)$ 的矩阵元衰和 $O_{r j}^{[n]}$ 衰达式的系数方程}

我们赋于杨盘标号以一定的意义,用 $S_{x y}^{[l]}$ 表示杨盘. $x$ 是数字 $n$ 的列指标, $y$ 是 $n-1$ 的 列指标. 我们规定杨盘列的次序是从右向左递增的. 除掉比 [ $\lambda]$ 多一列的那些杨盘要排在表 的最右之外,其余都按照 $x$ 和 $y$ 从左到右从上到下递增的次序排列成表. 例如, $[\lambda]=\left[2^{a} 1^{b}\right]$, 可得表 1 .

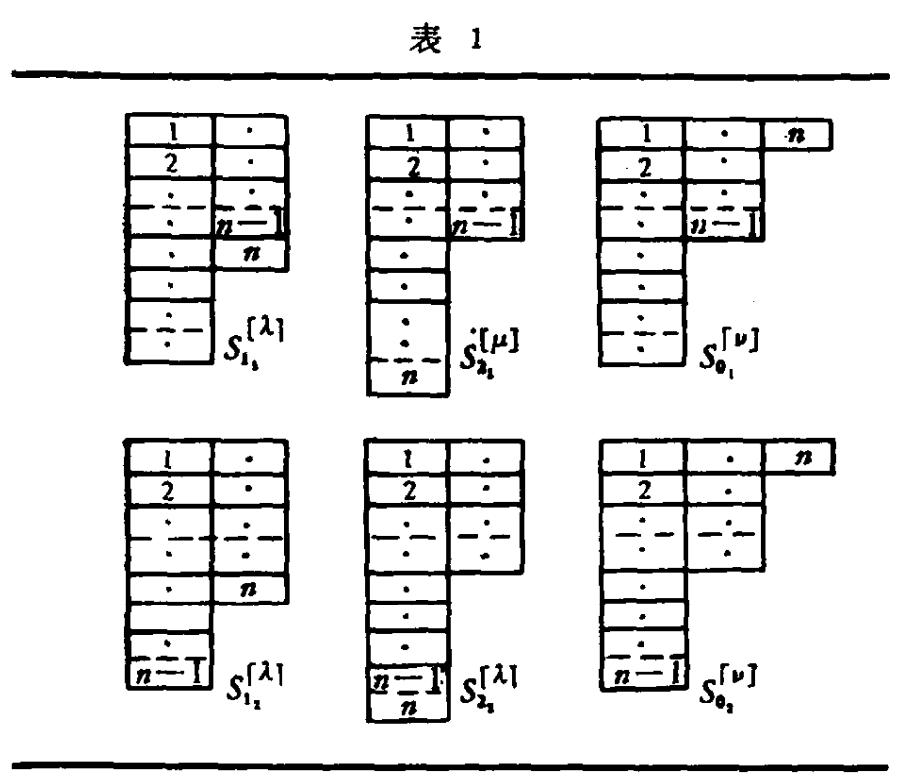

按照同样的次序, 将 $(n-1, n)$ 的 Young-Yamanouchi-Rutherford 矩阵 ${ }^{[2]}$ 也排列成表可得 (表 2 ).

本文1982 年9月3 日收到. 
表 2a

\begin{tabular}{|c|c|c|}
\hline$[\lambda]$ & {$[\mu]$} & {$[\boldsymbol{\nu}]$} \\
\hline$U_{1,1}^{[\lambda]}(n-1, n)$ & $U_{2_{1}{ }_{1}{ }_{1}}^{\mathrm{L \mu}}(n-1, n)$ & $U_{0,1}^{L+1}(n-1, n)$ \\
\hline$U_{\left.t_{2}\right]_{2}}^{[2]}(n-1, n)$ & $U_{z_{2^{2}}}^{[\mu]}(n-1, n)$ & $U_{0_{2} 0_{2}}^{[\nu]}(n-1, n)$ \\
\hline
\end{tabular}

表 $2 b$

\begin{tabular}{c|c|c}
\hline$[\lambda]$ & {$[\mu]$} & {$[\nu]$} \\
\hline-1 & $\frac{-1}{b+3}$ & $\frac{1}{a-1}$ \\
$\frac{1}{b+1}$ & -1 & $\frac{1}{a+b+1}$ \\
\hline
\end{tabular}

在此种情况下确定 $O_{r s}^{[\lambda] ~}$ 系数的方程组是

$$
\begin{aligned}
& O=1-\frac{1}{a-1} C_{1}+\frac{1}{a+b+1} C_{2}, \\
& O=1-\frac{1}{b+3} C_{1}-C_{2}, \\
& 1=\frac{\theta^{1}}{\theta}\left(1-C_{1}+\frac{1}{b+1} C_{2}\right),
\end{aligned}
$$

显然,解这样的方程组没有任何困难,但是我们感兴趣的是高对称型.

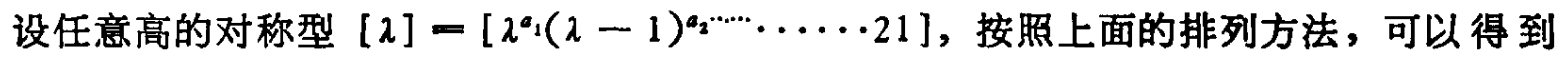
$(n-1, n)$ 的矩阵元表 (表 3).

表 3

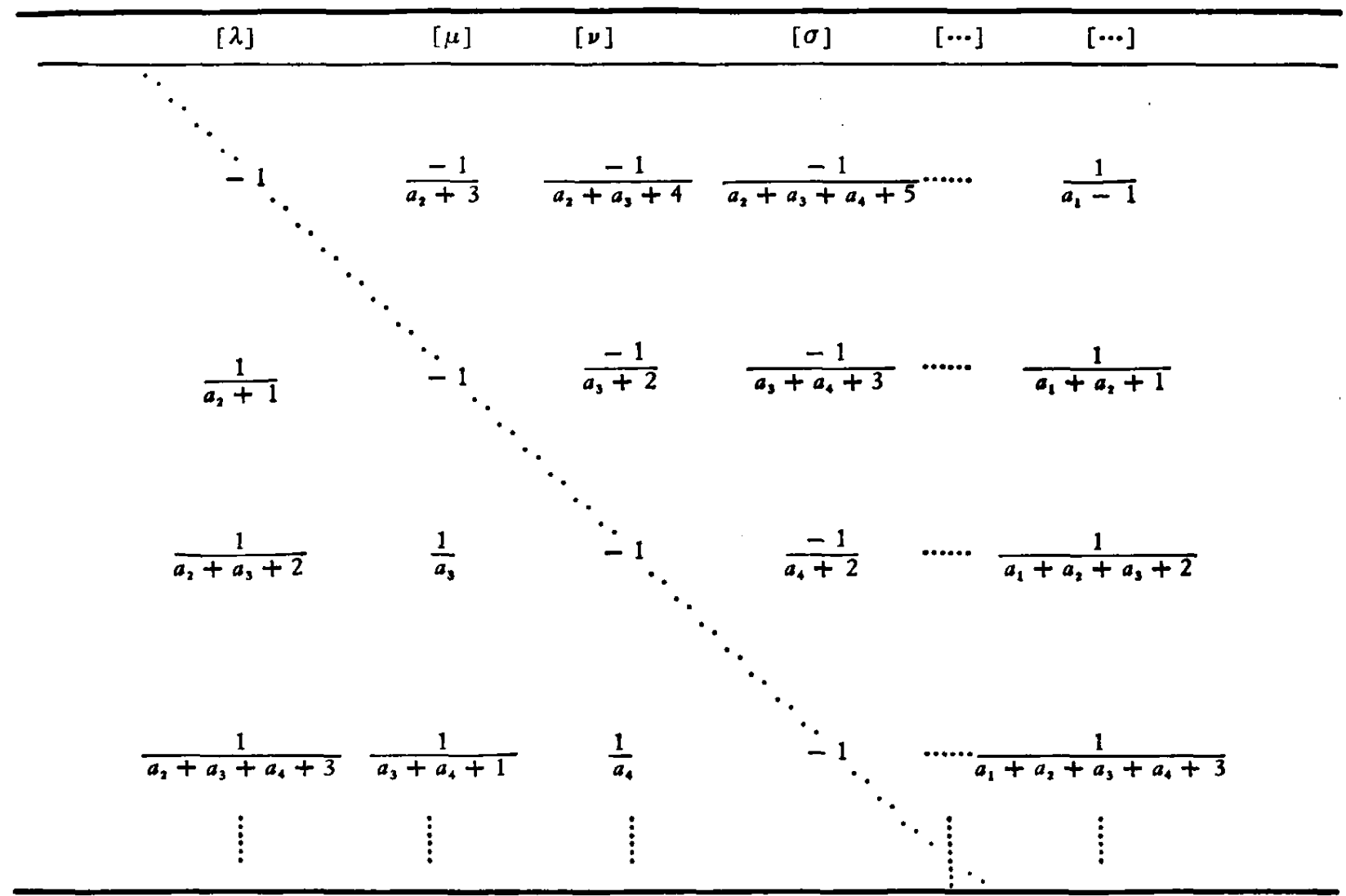

系数方程组将是 (暂不讨论 $\frac{\theta^{\prime}}{\theta}$ 的方程)

$$
0=\frac{1}{a_{1}-1} C_{1}^{[\lambda]}+\frac{1}{a_{1}+a_{2}+1} C_{2}^{[\lambda]}+\frac{1}{a_{1}+a_{2}+a_{3}+2} C_{3}^{[\lambda]}+\cdots
$$




$$
\begin{aligned}
& \quad+\frac{1}{a_{1}+\cdots+a_{2}+\lambda-1} C_{\lambda}^{[\lambda]}, \\
& 0-1-\frac{1}{a_{2}+3} C_{1}^{[\lambda]}-C_{2}^{[\lambda]}+\frac{1}{a_{3}} C_{3}^{[\lambda]}+\frac{1}{a_{3}+a_{4}+1} C_{4}^{[\lambda]}+\cdots \\
& \quad+\frac{1}{a_{3}+\cdots+a_{2}+\lambda-3} C_{2}^{[\lambda]}, \\
& 0-1-\frac{1}{a_{2}+a_{3}+4} C_{1}^{[\lambda]}-\frac{1}{a_{3}+2} C_{2}^{[\lambda]}-C_{3}^{[\lambda]}+\frac{1}{a_{4}} C_{1}^{[\lambda]}+\cdots \\
& \quad+\frac{1}{a_{4}+\cdots+a_{\lambda}+\lambda-4} C_{2}^{[\lambda]}, \\
& 0=1-\frac{1}{a_{2}+a_{3}+\cdots+a_{2}+\lambda+1} C_{1}^{[\lambda]} \\
& \quad-\frac{1}{a_{3}+a_{4}+\cdots+a_{\lambda}+\lambda-1} C_{2}^{[\lambda]}-\cdots \cdots-C_{\lambda}^{[\lambda]} .
\end{aligned}
$$

式中 $\lambda$ 是 $[\lambda]$ 中最大的数字.

直接解方程组是个辣手的问题,而且对称类型愈高困难越大. 下面将给出它的解.

\section{三、方程组的解和因形规则}

作变换, 令 $X_{1}=\sum_{m=1}^{l} a_{m}+\lambda, X_{i}=\sum_{m=i}^{l} a_{m}+[\lambda-(i-1)]$, 则系数方程组 (2)变为

$$
\begin{aligned}
& 1+\frac{C_{1}^{[\lambda]}}{X_{K}-X_{2}-2}+\sum_{n=2}^{l} \frac{C_{n}^{[\lambda]}}{X_{K}-X_{n+1}-1}=0 \text { (当 } K=1 \text { 时), } \\
& 1-\frac{C_{1}^{[\lambda]}}{X_{2}-X_{K+1}+2}-\sum_{n=2 \neq K}^{l} \frac{C_{n}^{[\lambda]}}{X_{n+1}-X_{K+1}+1}=0(K=2 、 3 、 \cdots \lambda) .
\end{aligned}
$$

可以证明*, (3) 式的解为

$$
\begin{aligned}
& C_{1}^{[\lambda]}-C_{1}^{[2-1]} \frac{\left(X_{2}-X_{\lambda+1}+2\right)}{\left(X_{2}-X_{\lambda+1}+1\right)}=-\left(X_{1}-X_{2}-2\right) \frac{\prod_{m=2}^{2}\left(X_{2}-X_{m+1}+2\right)}{\prod_{m=2}^{2}\left(X_{2}-X_{m+1}+1\right)}, \\
& \underset{\substack{n<\lambda) \\
(n<1)}}{C_{n}^{[\lambda-1]}} \frac{\left(X_{n+1}-X_{\lambda+1}+1\right)}{\left(X_{n+1}-X_{\lambda+1}\right)}=\frac{\left(X_{1}-X_{n+1}-1\right)}{\left(X_{2}-X_{n+1}+1\right)} \frac{\prod_{m=2}^{l}\left(X_{n+1}-X_{m+1}+1\right)}{\prod_{m=2 \neq n}^{l}\left(X_{n+1}-X_{m+1}\right)} .
\end{aligned}
$$

式中 [ $\lambda-1]$ 表示杨图 [ $\lambda]$ 去掉最左一列后剩下的杨图. (4) 式还给出了解的递推性质.

为了更方便地应用解 (4), 我们给出简明的图形规则. 为此,我们定义: 杨图 $[\lambda]$ 中第 $i$ 列最末格到第 $i$ 列最末格的轴距为第 $i$ 列到第 $j$ 列的“梯距”, 用 $T_{i j}^{(\mu)}$ 表示. 则 (4) 式可表为

*可用第二归纳法证. 在此只须将(4)式代入(3)式即可验证. 


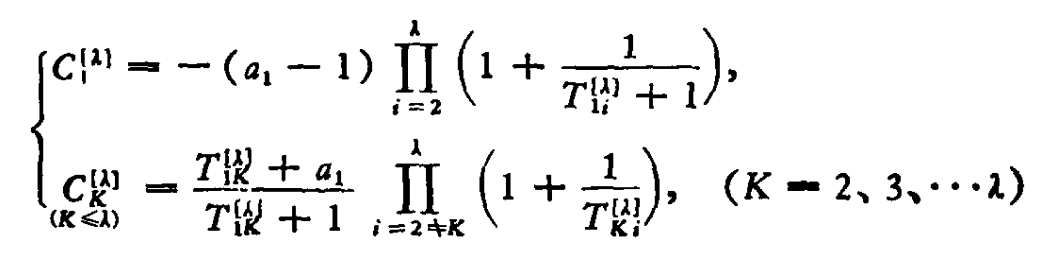

同样还可以得到

$$
\left\{\begin{array}{l}
C_{K}^{[\lambda]}=a_{1}\left(1+\frac{2}{T_{i(m+1)}^{[\lambda]}}\right)^{\frac{1}{2}} \cdot \prod_{i=2 \neq m+1}^{\lambda}\left(1+\frac{1}{T_{l_{i}}^{[\lambda}}\right),(K-\lambda+1, \cdots 2 \lambda-1) \\
\left(\frac{\theta}{\theta^{\prime}}\right)^{i \lambda]}=a_{1} \prod_{i=2}^{\lambda}\left(1+\frac{1}{T_{1 i}^{[\lambda}}\right) .
\end{array}\right.
$$

任意给定的 [ $\lambda]$ 画出所对应的杨图,很容易写出 $O_{r s}^{[\lambda]}$ 的全部系数。

例: $[\lambda]=\left[4^{a_{1}} 3^{a_{2}} 2^{a_{3}} 1^{a_{4}}\right]$

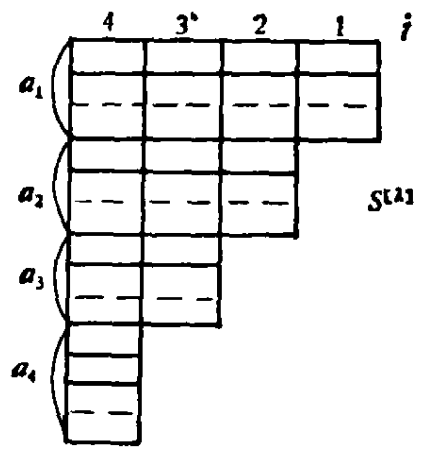

图 1

$$
\begin{aligned}
C_{1}^{[\lambda]} & =-\left(a_{1}-1\right)\left(1+\frac{1}{T_{12}^{[\lambda]}+1}\right)\left(1+\frac{1}{T_{13}^{[\lambda]}+1}\right)\left(1+\frac{1}{T_{14}^{[\lambda]}+1}\right) \\
& =-\frac{\left(a_{1}-1\right)\left(a_{2}+3\right)\left(a_{2}+a_{3}+4\right)\left(a_{2}+a_{3}+a_{4}+5\right)}{\left(a_{2}+2\right)\left(a_{2}+a_{3}+3\right)\left(a_{2}+a_{3}+a_{4}+4\right)}, \\
C_{6}^{[\lambda]} & =a_{1}\left(1+\frac{2}{T_{13}^{[\lambda]}-1}\right)^{\frac{1}{2}}\left(1+\frac{1}{T_{12}^{[\lambda]}}\right)\left(1+\frac{1}{T_{14}^{[\lambda]}}\right) \\
& =a_{1}\left(\frac{a_{2}+a_{3}+3}{a_{2}+a_{3}+1}\right)^{\frac{1}{2}} \frac{\left(a_{2}+2\right)\left(a_{2}+a_{3}+a_{4}+4\right)}{\left(a_{2}+1\right)\left(a_{2}+a_{3}+a_{4}+3\right)} \\
\left(\frac{\theta}{\theta^{\prime}}\right)^{[\lambda]} & =a_{1}\left(1+\frac{1}{T_{12}^{[\lambda]}}\right)\left(1+\frac{1}{T_{13}^{[\lambda]}}\right)\left(1+\frac{1}{T_{14}^{[\lambda]}}\right) \\
& =\frac{a_{1}\left(a_{2}+2\right)\left(a_{2}+a_{3}+3\right)\left(a_{2}+a_{3}+a_{4}+4\right)}{\left(a_{2}+1\right)\left(a_{2}+a_{3}+2\right)\left(a_{2}+a_{3}+a_{4}+3\right)} .
\end{aligned}
$$

\section{结束语}

这种方法可以直接推广到半正则表示,也很有希望成为处理大群有关问题的突破口.

[1] 孙共洲, 北京大学学报, 2(1962), 134.

[2] Rutherford, D. E., Substitutional Analysis., Edinburgh, 1948, 49.

[3]庄勤, 高能物理与核物理, 6 (1982), 3:280。 